\title{
Coherent Light Scattering from a Two-Dimensional Mott Insulator
}

\author{
Christof Weitenberg, ${ }^{1}$ Peter Schauß, ${ }^{1}$ Takeshi Fukuhara, ${ }^{1}$ Marc Cheneau, ${ }^{1}$ Manuel Endres, ${ }^{1}$ \\ Immanuel Bloch, ${ }^{1,2}$ and Stefan Kuhr ${ }^{1,3, *}$ \\ ${ }^{1}$ Max-Planck-Institut für Quantenoptik, Hans-Kopfermann-Straße 1, 85748 Garching, Germany \\ ${ }^{2}$ Ludwig-Maximilians-Universität, Schellingstraße 4/II, 80799 München, Germany \\ ${ }^{3}$ Department of Physics, SUPA, University of Strathclyde, Glasgow G4 ONG, United Kingdom \\ (Received 18 February 2011; revised manuscript received 24 March 2011; published 23 May 2011)
}

We experimentally demonstrate coherent light scattering from an atomic Mott insulator in a twodimensional lattice. The far-field diffraction pattern of small clouds of a few hundred atoms was imaged while simultaneously laser cooling the atoms with the probe beams. We describe the position of the diffraction peaks and the scaling of the peak parameters by a simple analytic model. In contrast to Bragg scattering, scattering from a single plane yields diffraction peaks for any incidence angle. We demonstrate the feasibility of detecting spin correlations via light scattering by artificially creating a one-dimensional antiferromagnetic order as a density wave and observing the appearance of additional diffraction peaks.

Ultracold atoms in optical lattices have become a useful tool to simulate static phases and the dynamical responses of quantum many-body systems [1]. Recent interest has focused on reaching sufficiently low temperatures and entropies to observe magnetically ordered quantum phases [2]. In this context, light scattering has been proposed as a new tool to detect these quantum correlations. Spin correlations could be mapped onto correlations of scattered light [3] or be detected via diffraction peaks from the additional scattering planes for spin-dependent probe light [4]. Light scattering would allow us to measure the temperature of fermions in an optical lattice [5] or the density fluctuations across the superfluid-to-Mott-insulator (MI) transition $[6,7]$. Since the amount of scattered light is usually very small, several proposals involve a cavity for the detection [8]. Without cavities, elastic Bragg scattering has been used to demonstrate the long-range periodic order of thermal atoms in an optical lattice despite very low filling factors $[9,10]$. It allowed the measurement of a change of the lattice constant from the backaction of the atoms [9,10], their localization dynamics [11], and their temperature [10]. Bragg scattering was also studied in a fardetuned one-dimensional lattice [12].

Here we show coherent light scattering from an atomic MI in a two-dimensional square lattice structure. Scattering from a 2D geometry differs significantly from usual Bragg scattering, because the momentum transfer needs to be an integer multiple of a reciprocal lattice vector only within the plane. Therefore, diffraction peaks appear for any angle of the incoming beam, which is experimentally more convenient. In our setup, we use five probe beams in a molasses configuration that simultaneously laser cool the atoms. Each of these molasses beams yields distinct diffraction peaks in the far-field images. We quantitatively compared the diffraction patterns with model calculations and confirmed the coherent nature of the scattering process. We artificially prepared $1 \mathrm{D}$ antiferromagnetic (AFM) order as a density wave and observed additional diffraction peaks, thus demonstrating the usability of light scattering for the detection of global spin correlations.

We begin by introducing a simple analytic 1D model to illustrate the underlying physics. Atoms on a 1D lattice (lattice spacing $a_{\text {lat }}=\lambda_{\text {lat }} / 2, \lambda_{\text {lat }}$ is the lattice wavelength) are driven by an incoming light field (wavelength $\lambda$ ) from the $x$ direction [Fig. 1(a)] with wave vector $\mathbf{k}_{i}=k \mathbf{e}_{x}$, where $k=2 \pi / \lambda$ and $\mathbf{e}_{x}$ is the unit vector along $x$. The scattered light is detected at a point $\mathbf{r}$, defined by the angle $\theta$ with the $z$ axis, such that $\mathbf{r}=r \mathbf{e}_{r}=r \sin \theta \mathbf{e}_{x}+$ $r \cos \theta \mathbf{e}_{z}$. The position of the $l$ th atom is $\mathbf{x}_{l}=l a_{\text {lat }} \mathbf{e}_{x}$ and its distance $r_{l}$ to the detection point is in far-field approximation $r_{l}=\left|\mathbf{r}-\mathbf{x}_{l}\right| \approx r-\mathbf{x}_{l} \cdot \mathbf{e}_{r}$. In our model, each atom emits a spherical wave, which at the detection point can be written as

$$
F_{l}\left(r_{l}\right)=f \frac{e^{i k r_{l}}}{r_{l}} e^{i \delta_{l}} \approx f \frac{e^{i k r}}{r} e^{-i \mathbf{K} \cdot \mathbf{x}_{l}} .
$$

Here, $f$ denotes the coherently scattered field amplitude, $\delta_{l}=\mathbf{k}_{i} \cdot \mathbf{x}_{l}$ is the phase imprinted by the incoming light field, and $\mathbf{K}=\mathbf{k}_{o}-\mathbf{k}_{i}$ with the wave vector $\mathbf{k}_{o}=k \mathbf{e}_{r}$ in the observed direction. The differential cross section $\frac{d \sigma}{d \theta}(\mathbf{K}) \propto\left|\sum_{l} e^{-i \mathbf{K} \cdot \mathbf{x}_{l}}\right|^{2}$ is obtained by summing over the field amplitudes from all $N_{x}$ atoms. As a result, we obtain the angular dependence of the scattered light field,

$$
\frac{d \sigma}{d \theta}(\theta) \propto \frac{\sin ^{2}\left[k a_{\mathrm{lat}}(\sin \theta-1) N_{x} / 2\right]}{\sin ^{2}\left[k a_{\mathrm{lat}}(\sin \theta-1) / 2\right]},
$$

with distinct maxima when the field amplitudes of neighboring atoms interfere constructively, i.e., $\mathbf{K} \cdot\left(\mathbf{x}_{l}-\mathbf{x}_{l+1}\right)=$ $(2 \pi) n$, where $n$ is an integer that denotes the diffraction order. The height of the diffraction peak is proportional to 


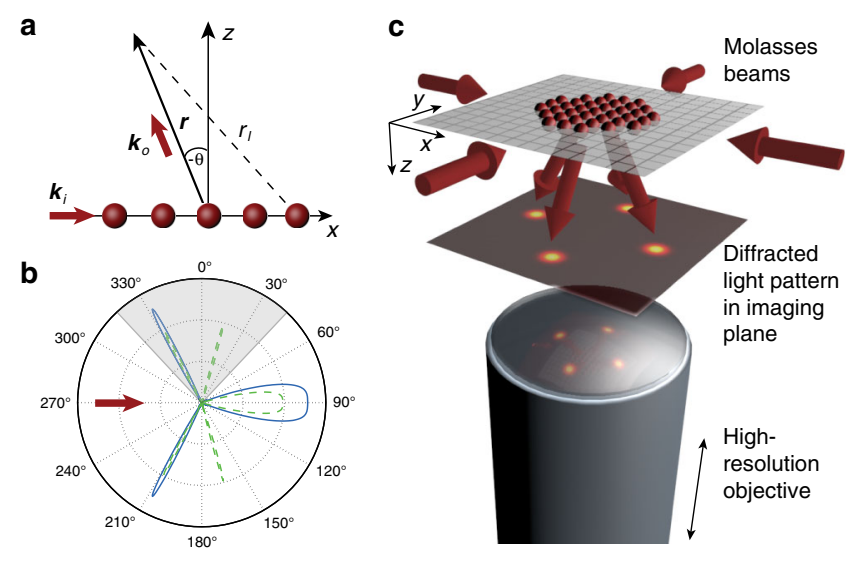

FIG. 1 (color online). Schematics of light scattering. (a) Light diffraction from atoms in a 1D lattice. An incoming wave with wave vector $\mathbf{k}_{i}$ is diffracted under an angle $\theta$. (b) Resulting differential scattering cross section $\frac{d \sigma}{d \theta}(\theta)$, as given by Eq. (2) for our experimental parameters. Shown are the cases for unity filling of the lattice and atom number $N=16$ (solid blue curve) and for a $z$-Néel AFM along one direction $(N=28)$, from which only one spin component is detected (dashed green curve). The gray shaded area indicates the opening angle of our imaging system. (c) Experimental setup. Atoms in a 2D optical lattice are illuminated with four in-plane molasses beams. In situ and farfield diffraction images are recorded with a high-resolution optical imaging system.

$N_{x}^{2}$ whereas the peak width scales with $1 / N_{x}$. The angles $\theta_{n}$, under which the diffraction maxima can be observed, are given by

$$
\sin \theta_{n}=1+n \frac{\lambda}{a_{\text {lat }}} .
$$

The trivial case $n=0$ gives the forward scattered light $\left(\theta_{0}=90^{\circ}\right)$, independent of $a_{\mathrm{lat}}$ and $\lambda$. For our experimental parameters $\left(\lambda=780 \mathrm{~nm}, a_{\mathrm{lat}}=532 \mathrm{~nm}\right)$, Eq. (3) can be additionally fulfilled only for $n=-1$, yielding the corresponding minus first diffraction order at $\theta_{-1}=-27.8^{\circ}$ and $207.8^{\circ}$. These two out of plane scattered waves ensure the momentum conservation in the $z$ direction. Figure 1(b) (solid blue curve) shows a polar plot of $\frac{d \sigma}{d \theta}(\theta)$, displaying the forward scattered light and the two peaks, one of which is captured by our imaging system (gray shaded region). If only every second lattice site is occupied (e.g., after removing one spin component in an antiferromagnetically ordered sample), the periodicity of the system is doubled. In this case, there are two possible diffraction orders (in the upper half plane) at $\theta_{-1}^{\mathrm{AFM}}=15.5^{\circ}$ and $\theta_{-2}^{\mathrm{AFM}}=\theta_{-1}=-27.8^{\circ} \quad$ [dashed green curve in Fig. 1(b)].

In our experiment, we prepared 2D MIs of ${ }^{87} \mathrm{Rb}$ atoms in an optical lattice. The atoms were confined in a single antinode of a vertical standing wave. Two pairs of horizontal laser beams provided the square lattice structure (see Ref. [13]). In order to detect the atoms in the optical lattice, the lattice depth was increased to $\sim 300 \mu \mathrm{K}$, thereby freezing the atom distribution. The atoms where

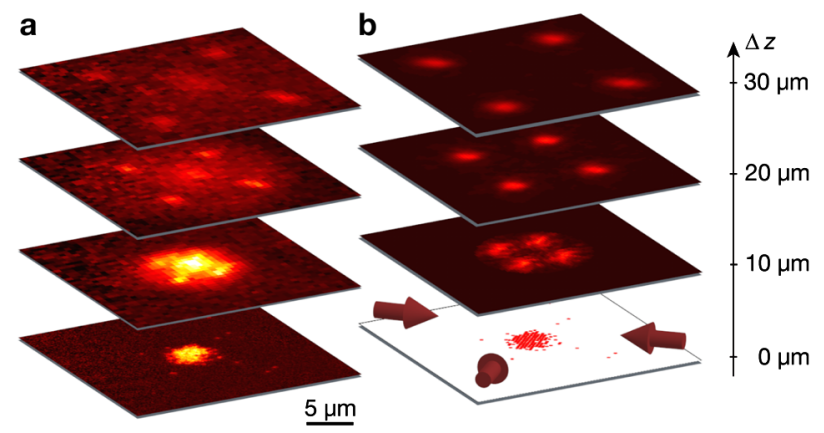

FIG. 2 (color online). Light scattering from a 2D MI. (a) Experimental images from the same atomic sample for four different distances $\Delta z$ of the focal plane with respect to the atom position. The lowest image shows the in situ atom number distribution, whereas the upper image shows the far-field diffraction pattern. (b) Simulated diffraction patterns obtained from a 2D numerical model (see text for details), using the reconstructed atom distribution (lower image) from (a). Red arrows indicate the directions of the optical molasses beams.

then illuminated with an optical molasses, which consisted of two pairs of retroreflected laser beams oriented along the horizontal lattice axes [see Fig. 1(c)]. A fifth molasses beam entered from the reverse direction of the imaging system [not shown in Fig. 1(c)]. The molasses was reddetuned with respect to the free space resonance by $45 \mathrm{MHz}$ and the total scattering rate was $60 \mathrm{kHz}$. We detected the fluorescence photons with a high-resolution microscope objective with half opening angle $\alpha=43^{\circ}$. The objective can be moved in the $z$ direction by a distance $\Delta z<100 \mu \mathrm{m}$ between the atom position and the focal plane within $50 \mathrm{~ms}$ using a piezoscanning device.

Our experimentally obtained diffraction images are shown in Fig. 2(a) for four different distances $\Delta z$ and an illumination time of $200 \mathrm{~ms}$ for each image. For $\Delta z=0$ we observed the in situ atom number distribution, consisting in this case of 147 atoms in a MI shell with unit occupancy and diameter of $6 \mu \mathrm{m}$. For larger $\Delta z$, we observe the buildup of the far-field distribution with distinct diffraction peaks. We compared the experimental data with a numerical calculation of $\frac{d \sigma}{d \theta}(\mathbf{K})$ using the actual atom distribution of the image at $\Delta z=0$ [Fig. 2(b)]. For this purpose, we coherently summed over all spherical waves $F_{l}\left(r_{l}\right)$ emitted by the atoms with phases $\delta_{l}$ given by the incident driving fields. Our model assumes that all four horizontal molasses beams are diffracted independently. A spherical wave for the emission pattern is used because the different local polarizations in the molasses result in all possible orientations of the atomic dipole. The calculated far-field distribution is in good qualitative agreement with the experimental images. Our simulation only includes the coherently scattered light, whereas the experimental data also show a significant incoherent background.

For a more quantitative analysis, we recorded diffraction patterns of MIs for different atom numbers 
[Figs. 3(a) and 3(c)]. We evaluated cuts (angular sectors of width $4^{\circ}$ ) through the diffraction peaks and the background signal [see Fig. 3(c)] and applied an appropriate coordinate transformation in order to obtain the angular distribution $\frac{d \sigma}{d \theta}(\theta)$, as shown in Fig. 3(d). We fitted the resulting peaks with a Gaussian (height $A, 1 / \sqrt{e}$ width $w$, center position $\theta_{-1}$, and offset fixed at the background value). The peak position, averaged over all experimental runs, is $\left|\theta_{-1}\right|=$ $27.4(6)^{\circ}$, in excellent agreement with the expected value of $\left|\theta_{-1}\right|=27.8^{\circ}$. The error is dominated by the systematic uncertainty of $\pm 1 \mu \mathrm{m}$ in the determination of $\Delta z$. The peak height scales quadratically with the atom number [Fig. 3(e)], illustrating the coherent nature of the diffrac-
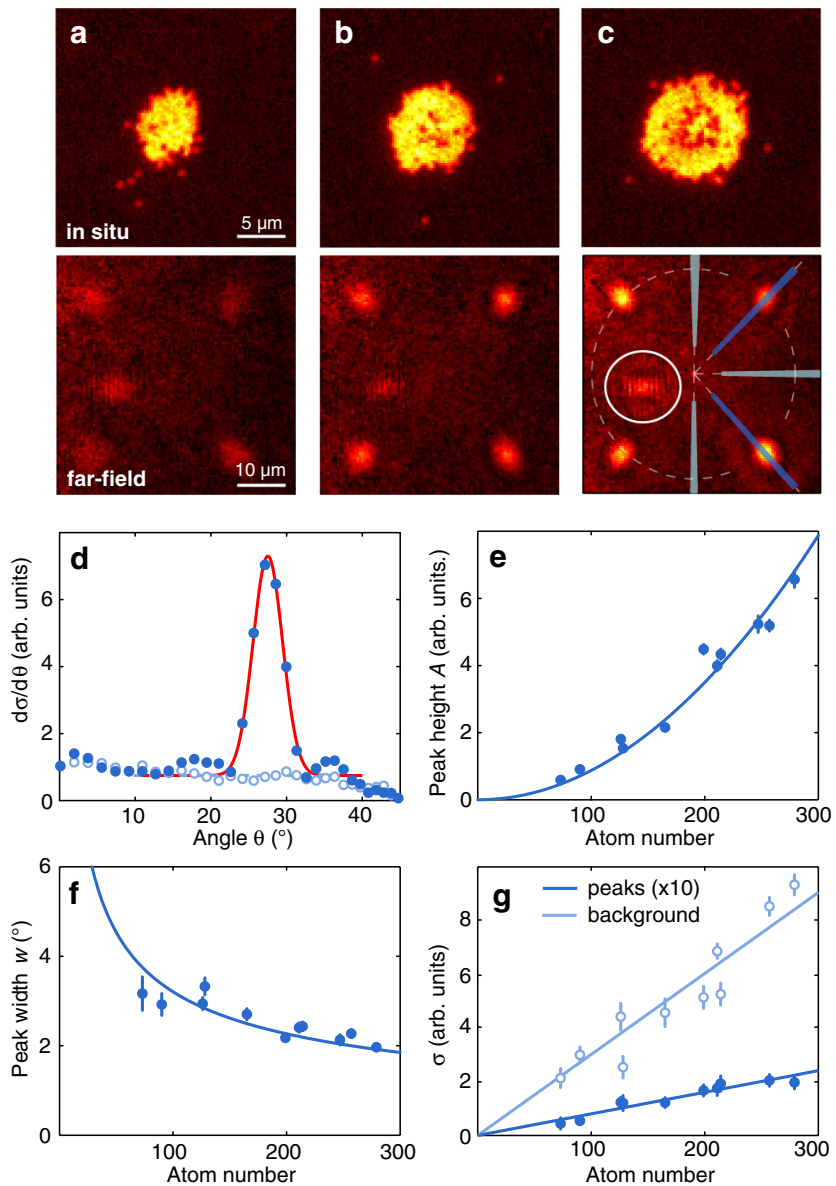

FIG. 3 (color online). Analysis of the diffraction patterns. (a)-(c) In situ images ( $N=126,199$, and 279 atoms) and the corresponding far-field diffraction pattern $(\Delta z=40 \mu \mathrm{m})$ from the same experimental run, with illumination time $500 \mathrm{~ms}$ each. (d) Angular distribution of the differential scattering cross section obtained from cuts in (c) through the diffraction peaks (dark blue, filled symbols) and the background signal (light blue, open symbols). The peak is fitted with a Gaussian (solid red line). (e) Peak height $A$ versus $N$ together with a quadratic fit. (f) Peak width $w$ versus $N$, with a fit to $w \propto 1 / \sqrt{N}$. (g) Total background signal $\sigma_{b}$ (light blue, open symbols) as obtained from the constant background in (d) and total signal in the peaks $\sigma_{p}$ (dark blue, filled symbols, scaled by a factor of 10) obtained from the fits to the diffraction peaks (see text). tion peak. The peak width scales as $w \propto 1 / \sqrt{N}$ [Fig. 3(f)], in agreement with the result from the 1D model [Eq. (2)], assuming $N_{x}=\sqrt{N}$ atoms in one dimension.

There are several mechanisms that lead to a background outside the diffraction peaks. The first mechanism is the deviation from integer occupation of the lattice sites caused by density fluctuations in the system [6]. The second mechanism is the spread of the atoms in their potential wells, which reduces the peak height via the Debye-Waller factor. In our case, however, the dominant mechanism is inelastic scattering. While in a two-level picture, the scattering is expected to be completely coherent for our large detuning and small saturation parameter [14], the scattering from a molasses configuration constantly changes the magnetic sublevels, thus preventing interference of the scattered light. Scattering processes that change the vibrational level are negligible due to the small Lamb-Dicke parameter. We used the far-field images to extract the power scattered into the detected diffraction peaks and the power scattered into the background. In the cuts outside the diffraction peaks, we found a constant background $\left(\frac{d \sigma}{d \theta}\right)_{b}$ and calculated the total incoherent scattering cross section $\sigma_{b}=4 \pi\left(\frac{d \sigma}{d \theta}\right)_{b}$, assuming an isotropic intensity distribution. The total scattering cross section from the five molasses beams can be estimated as $\sigma_{p} \approx$ $10 \sin \left(\theta_{-1}\right) 2 \pi A w^{2} \cos \left(\theta_{-1}\right)$. The factor $\cos \left(\theta_{-1}\right)$ accounts for the ellipticity of the diffraction peaks due to the effective ellipticity of the atomic cloud, when viewed under the angle $\theta_{-1}$. Figure $3(\mathrm{~g})$ shows $\sigma_{b}$ and $\sigma_{p}$, which both scale linearly with the number of atoms. From the slopes, we find a fraction $f_{p}=\sigma_{p} /\left(\sigma_{b}+\sigma_{p}\right) \approx 3 \%$ of the power scattered into the detected peaks. Only about $20 \%$ of the coherently scattered light is diffracted into these peaks, while the forward scattered part is not detected here.

In addition to the four diffraction peaks from the horizontal molasses beams, a fifth weaker peak is clearly visible in the center left part of the far-field images [see white circle in Fig. 3(c)]. This peak results from the diffraction of the molasses beam which is shone in from the direction of the imaging system. It shows that our single plane of a few hundred atoms in the optical lattice acts as a "mirror" for the incoming laser beam.

Finally, we demonstrated that light scattering can be used for the detection of spin correlations. As an example, we created a 1D $z$-Néel AFM order along the $x$ direction of the lattice using our recently demonstrated single-site addressing technique [15]. We sequentially flipped all atomic spins in every second row of the lattice from $F=1$ to $F=2$ before we applied a resonant laser that removed all atoms in $F=2$. Figures 4(a) and 4(b) show the resulting fluorescence image in the focal plane together with the reconstructed atom number distribution. The corresponding experimental and theoretical diffraction patterns are displayed in Figs. 4(c) and 4(d). The two predicted diffraction peaks of -1 st and -2 nd order along $x$ are clearly 

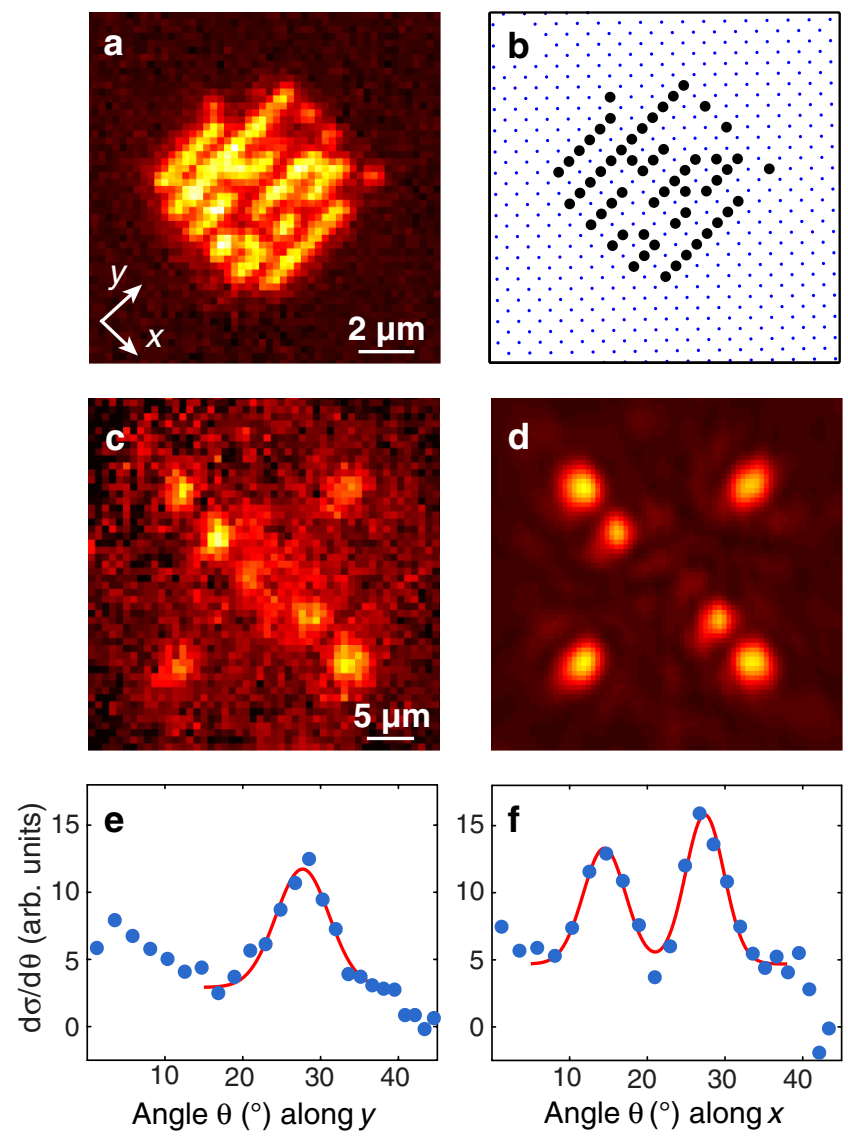

FIG. 4 (color online). Light scattering for 1D antiferromagnetic order in the density. (a) The atoms in every second row were removed. (b) Reconstructed atom number distribution from (a). (c) Resulting far-field image $(\Delta z=25 \mu \mathrm{m})$ with two diffraction orders in the $x$ direction. (d) Simulated diffraction pattern using the atom number distribution of (b). (e),(f) Angular distribution of the differential scattering cross section obtained from cuts along the $x$ and $y$ directions together with Gaussian fits.

visible in the experimental picture, although our atomic sample consisted of only 57 atoms. We obtain the usual peak position of $\left|\theta_{-1}^{y}\right|=27.7(6)^{\circ}$ along the $y$ direction, whereas the two peaks along $x$ are found at $\left|\theta_{-1}^{x}\right|=$ 14.5(6) ${ }^{\circ}$ and $\left|\theta_{-2}^{x}\right|=27.4(6)^{\circ}$ [see Figs. 4(e) and 4(f)], in good agreement with the expected values. We prepared a 1D antiferromagnetic order because the additional diffraction peaks that would arise for a $2 \mathrm{D}$ antiferromagnetic order lie outside the opening angle of our imaging system. However, the position of the diffraction peaks could be varied in a $2 \mathrm{D}$ geometry by changing the angle of the incident beams. An alternative is to use shorter wavelength probe light, e.g., near resonant with the $5 S-6 P$ transition at $420 \mathrm{~nm}$ for ${ }^{87} \mathrm{Rb}$.

Our results could be extended to the study of various density $[6,7]$ or spin $[3,4]$ correlations in optical lattices. Most proposals suggest weak nondestructive probing, which restricts the signal to only a few photons per atom. In our alternative approach, we projected the correlations onto the density before the detection by means of an optical molasses, which yields a signal of thousands of photons per atom. This is possible for density correlations, as, e.g., the number squeezing in a MI can be mapped on the mean density by parity projection $[13,16]$. For spin correlations, we have demonstrated the feasibility of removing one spin state and observing the additional diffraction peaks from the density structure. This avoids spin selective coupling of the probe light to the atoms [4], which is incompatible with simultaneous laser cooling. In the 3D case, antiferromagnetic order allows scattering from an additional plane, but it requires careful alignment of the probe beam angle to match the Bragg condition. In contrast, light scattering from a 2D system yields additional peaks from the same incident beam, which is also convenient for the extraction of the spin correlation length from the relative height or width of the diffraction peaks. Finally, we note that the detection of spin correlations via light scattering does not rely on the high aperture of our imaging system. The diffraction peaks could also be detected in a restricted angular range.

We acknowledge funding by MPG, DFG, Stiftung Rheinland-Pfalz für Innovation, Carl-Zeiss Stiftung, EU (NAMEQUAM, AQUTE, Marie Curie Fellowship to M. C.), and JSPS (Postdoctoral Fellowship for Research Abroad to T.F.).

*stefan.kuhr@mpq.mpg.de

[1] I. Bloch, J. Dalibard, and W. Zwerger, Rev. Mod. Phys. 80, 885 (2008).

[2] M. Lewenstein, et al., Adv. Phys. 56, 243 (2007).

[3] J. Javanainen and J. Ruostekoski, Phys. Rev. Lett. 91, 150404 (2003); I. de Vega, J. I. Cirac, and D. Porras, Phys. Rev. A 77, 051804(R) (2008); K. Eckert et al., Nature Phys. 4, 50 (2007); J. S. Douglas and K. Burnett, Phys. Rev. A 82, 033434 (2010).

[4] T. A. Corcovilos et al., Phys. Rev. A 81, 013415 (2010).

[5] J. Ruostekoski, C. J. Foot, and A. B. Deb, Phys. Rev. Lett. 103, 170404 (2009).

[6] I. B. Mekhov, C. Maschler, and H. Ritsch, Phys. Rev. A 76, 053618 (2007).

[7] K. Lakomy, Z. Idziaszek, and M. Trippenbach, Phys. Rev. A 80, 043404 (2009); S. Rist, C. Menotti, and G. Morigi, Phys. Rev. A 81, 013404 (2010).

[8] I. B. Mekhov, C. Maschler, and H. Ritsch, Nature Phys. 3, 319 (2007); W. Chen, D. Meiser, and P. Meystre, Phys. Rev. A 75, 023812 (2007).

[9] G. Birkl et al., Phys. Rev. Lett. 75, 2823 (1995).

[10] M. Weidemüller et al., Phys. Rev. Lett. 75, 4583 (1995); M. Weidemüller et al., Phys. Rev. A 58, 4647 (1998).

[11] C. I. Westbrook et al., J. Mod. Opt. 44, 1837 (1997); G. Raithel et al., Phys. Rev. Lett. 78, 630 (1997).

[12] S. Slama et al., Phys. Rev. Lett. 94, 193901 (2005).

[13] J. F. Sherson et al., Nature (London) 467, 68 (2010).

[14] B. R. Mollow, Phys. Rev. 188, 1969 (1969).

[15] C. Weitenberg et al., Nature (London) 471, 319 (2011).

[16] W. S. Bakr et al., Science 329, 547 (2010). 